\title{
The Stochastic Resonance Phenomenon of Different Noises in Underdamped Bistable System
}

\author{
Shan Yang, ${ }^{1}$ Zening Fan, ${ }^{2}$ and Ruibin Ren $\mathbb{D}^{3}$ \\ ${ }^{1}$ Department of Computer and Software, Jincheng College of Sichuan University, Chengdu 611731, China \\ ${ }^{2}$ College of Mathematics, Sichuan University, Chengdu 610064, China \\ ${ }^{3}$ College of Mathematics, Southwest Jiaotong University, Chengdu 610031, China
}

Correspondence should be addressed to Ruibin Ren; airy_ren@163.com

Received 27 July 2020; Revised 31 December 2020; Accepted 28 January 2021; Published 11 February 2021

Academic Editor: Xiao-Ling Gai

Copyright (c) 2021 Shan Yang et al. This is an open access article distributed under the Creative Commons Attribution License, which permits unrestricted use, distribution, and reproduction in any medium, provided the original work is properly cited.

In this paper, the stochastic resonance (SR) phenomenon of four kinds of noises (the white noise, the harmonic noise, the asymmetric dichotomous noise, and the Lévy noise) in underdamped bistable systems is studied. By applying theory of stochastic differential equations to the numerical simulation of stochastic resonance problem, we simulate and analyze the system responses and pay close attention to stochastic control in the proposed systems. Then, the factors of influence to the SR are investigated by the Euler-Maruyama algorithm, Milstein algorithm, and fourth-order Runge-Kutta algorithm, respectively. The results show that the SR phenomenon can be generated in the proposed system under certain conditions by adjusting the parameters of the control effect with different noises. We also found that the type of the noise has little effect on the resonance peak of the output power spectrum density, which is not observed in conventional harmonic systems driven by multiplicative noise with only an overdamped term. Therefore, the conclusion of this paper can provide experimental basis for the further study of stochastic resonance.

\section{Introduction}

The concept of stochastic resonance (SR) was firstly proposed by Benzi et al. [1] in the 1980s to explain the periodic recurrence of ice ages on Earth. Since then, much attention has been paid to SR due to its potential applications in many fields [2-6].

In the past few years, many researchers focused the SR phenomenon of the overdamped systems $[2,4]$, while in recent years, researchers gradually shifted their views to the underdamped systems. The SR phenomenon in underdamped bistable system was firstly studied by Ray and Sengupta [7]; they analyzed the difference of the dependence of noise amplitude between underdamped bistable system and overdamped bistable system.

In fact, the bistable systems are very important on the noise effect of the nonlinear systems. Jia et al. [5] studied SR in bistable systems driven by additive and multiplicative white noise. Guo et al. [8] studied the instability probability density evolution in bistable systems driven by Gaussian noise and white noise, and obtained rich conclusions. Meanwhile, relevant theories have shown practical application significance in chemistry, physics, engineering, and other fields $[9,10]$. With the further study of stochastic phenomena, SR is gradually extended to multistable and more complex systems $[11,12]$. However, SR in bistable systems is still widely concerned by researchers due to its practical value.

On the other hand, the studies of early SR mechanism mainly focus on Gaussian white noise [13]. In recent years, however, some literatures have begun to focus on the effects of some non-Gaussian noises on SR of bistable systems [11, 14-17]. Wang et al. [11] studied the SR of the bistable system driven by simple harmonic noise. Zhang et al. [14] studied the stochastic resonance in the system driven by the Lévy noise and found interesting dynamic behaviors. Gingl et al. [15] studied the nondynamical SR with arbitrarily coloured noise, and Shen et al. [16] studied system driven by correlated non-Gaussian noise and Gaussian noise, while Neiman and Schimansky-Geier studied the SR in a bistable system driven by the harmonic noise. 
However, as the best of our knowledge, there is no detailed horizontal comparison of the dynamical effects among different noises in the available literature, and also there is a lack of simulation analyses of the complex system driven by nonwhite noise. As a matter of fact, most SR are difficult to be expressed in analytic form [17-20], especially for a wide range of situations in nonlinear systems. We noticed that the numerical simulation of SR is essentially numerically solving a stochastic differential equation. Hence, the numerical algorithm suitable for stochastic differential equations is more suitable for SR [21,22].

Therefore, the main goal of this paper is to focus on the control effects in an underdamped bistable system driven by four kinds of noises (the white noise, the harmonic noise, the asymmetric dichotomous noise, and the Lévy noise); meanwhile, we will provide the vivid numerical simulation analyses. Furthermore, since the harmonic noise can be generated by the white noise through the resonance subsystem, we would like to control the properties of harmonic noise by controlling the parameters of the resonance subsystem. Thus, the control of SR is realized.

The organization of this paper is as follows. Section 2 definitely introduces the system model and the definition of the four kinds of the noises and its parameters. The analyses of the effects of different noises on system output are given in Section 3 where we give the method to determine the number of simulations firstly. Finally, the conclusions are discussed in Section 4 .

\section{System Model}

We consider the undamped bistable system driven by four kinds of noise which is described by the following stochastic differential equation:

$$
\frac{d^{2} x}{d t^{2}}+\eta \frac{d x}{d t}=-\frac{\partial U(x)}{\partial x}+A \cos (\omega t+\theta)+\xi(t)
$$

where $\eta$ is the coefficient of the damping term, $U(x)$ is the system potential field, with $U(x)=-(1 / 2) a x^{2}+(1 / 4) b x^{4}, a$ $>0$ and $b>0$ are the two constants of potential field $U(x)$, $A, \omega, \theta$ are the amplitude, frequency, and phase of periodic driven force, respectively, and $\xi(t)$ is the noise; in this paper, we consider four types of noises which are the white noise, the harmonic noise, the asymmetric dichotomous noise, and the Lévy noise.

Firstly, we give a brief description of the four kinds of the noises as follows:

2.1. The White Noise. The white noise $\xi(t)$ is a stationary process with zero mean and constant power spectral density, respectively:

$$
\begin{aligned}
\langle\xi(t)\rangle & =0, \\
S(\omega) & =\frac{N_{0}}{2}, \quad \omega \in(-\infty,+\infty) .
\end{aligned}
$$

Besides, the white noise $\xi(t)$ has the following form of the second moment:

$$
\left\langle\xi(t) \xi\left(t^{\prime}\right)\right\rangle=2 \alpha \delta\left(t-t^{\prime}\right)
$$

Here, $\omega$ is the frequency of the white noise, $N_{0}$ is a constant independent with the frequency $\omega, \alpha$ is the noise intensity, and $\delta$ is the delta function.

2.2. The Harmonic Noise. The harmonic noise $\varepsilon(t)$ is a monochromatic noise commonly used in stochastic dynamics. It can be regarded as the output response of the resonant subsystem driven by Gaussian white noise $\xi(t)$ :

$$
\ddot{\varepsilon}(t)+\Gamma \dot{\varepsilon}(t)+\Omega^{2} \varepsilon(t)=\sqrt{2 \Theta \Gamma} \xi(t) .
$$

Here, $\Gamma, \Omega$, and $\Theta$ are the system parameters. Equation (3) determines the two-dimensional Ornstein-Uhlenbeck process $\varepsilon(t)$ and $\dot{\varepsilon}(t)$ with the power spectrum:

$$
S_{\varepsilon \varepsilon}(\omega)=\frac{\Theta \Gamma}{\omega^{2} \Gamma^{2}+\left(\omega^{2}-\Omega^{2}\right)^{2}},
$$

and the mean square displacements $\left\langle\varepsilon^{2}(t)\right\rangle=\Theta / \Omega^{2}$. From the expression function of simple harmonic noise power spectrum, it is not hard to know if $\Omega^{2}-\Gamma^{2} / 4 \geq 0$; the peak of power spectrum function should be at $\omega_{\mathrm{p}}=\sqrt{\Omega^{2}-\Gamma^{2} / 2}$. When $\Omega^{2}-\Gamma^{2} / 2<0$, the peak of power spectrum function should be at $\omega_{\mathrm{p}}=0$.

2.3. The Asymmetric Dichotomous Noise. We take $\xi(t)$ as the asymmetric dichotomous noise which consists of jumps between two values: $\{-a, k a\}$ with $a>0$ and $k>0$. The jumps follow, in time, according to the Poisson process. $k$ represents the asymmetric degree of the noise. When $k=1$, the noise becomes a symmetric noise. Let $\lambda$ and $\lambda^{\prime}$ be the transition rate from $-a$ to $k a$ and the reverse transition rate, respectively. Without loss of generality, we assume that

$$
\langle\xi(t)\rangle=\frac{k a \lambda-a \lambda^{\prime}}{\lambda+\lambda^{\prime}}=0 .
$$

Thus, we can obtain $k \lambda=\lambda^{\prime}$. Moreover, the correlation function of the asymmetric dichotomous noise is given by the following:

$$
\langle\xi(t) \xi(s)\rangle=D \gamma \exp \{-\lambda|t-s|\} .
$$

Here, $\gamma=\lambda+\lambda^{\prime}$ is the reverse of the correlation time $\tau$ of the asymmetric noise $\xi(t)$, and the definition of the strength of $\xi(t)$ is as follows:

$$
D=\frac{1}{2} \int_{-\infty}^{+\infty}\langle\xi(\tau) \xi(0)\rangle d \tau=\frac{k a^{2}}{\gamma} .
$$

Thus, we know that the noise strength $D$ is not independent, but is connected with the asymmetric degree $k$, the correlation time $\tau$, and value $a$. 


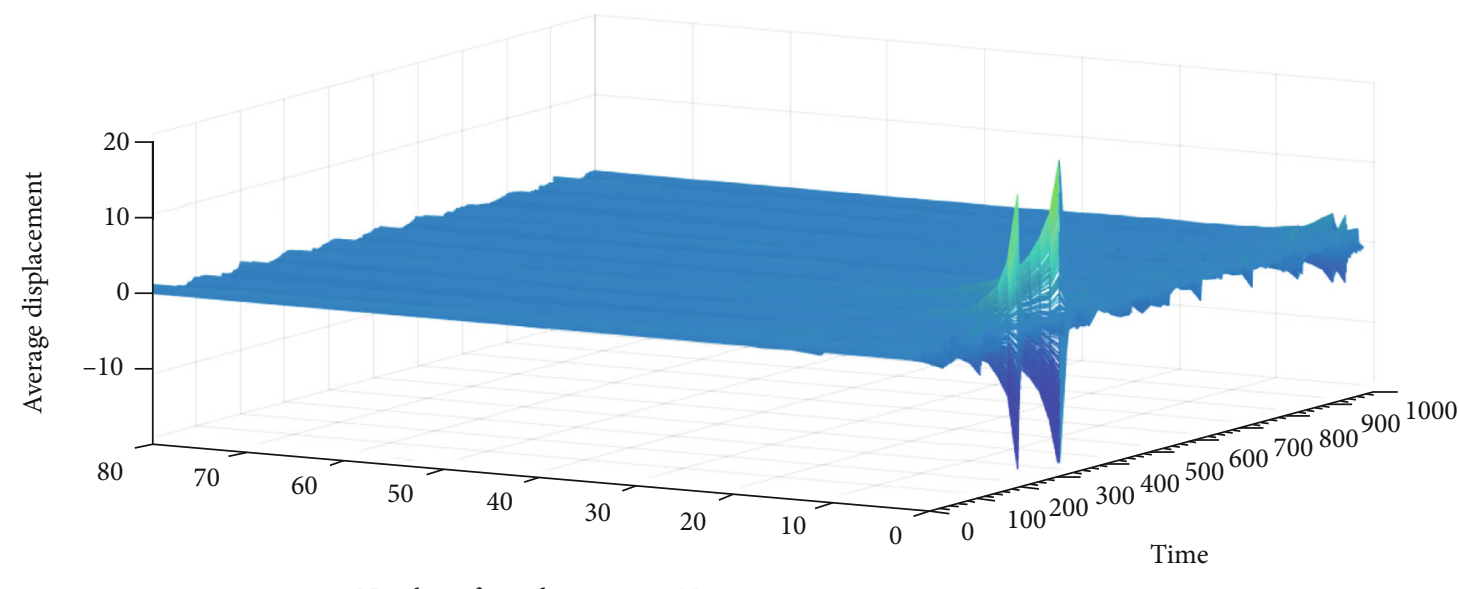

Number of simulation times $N_{i}$

FIGURE 1: The relationship between the output average displacement and simulation times of the system within time $T=1000$. The $x$-axis is the time, the $y$-axis is the number of simulation times, and the $z$-axis is the average displacement.

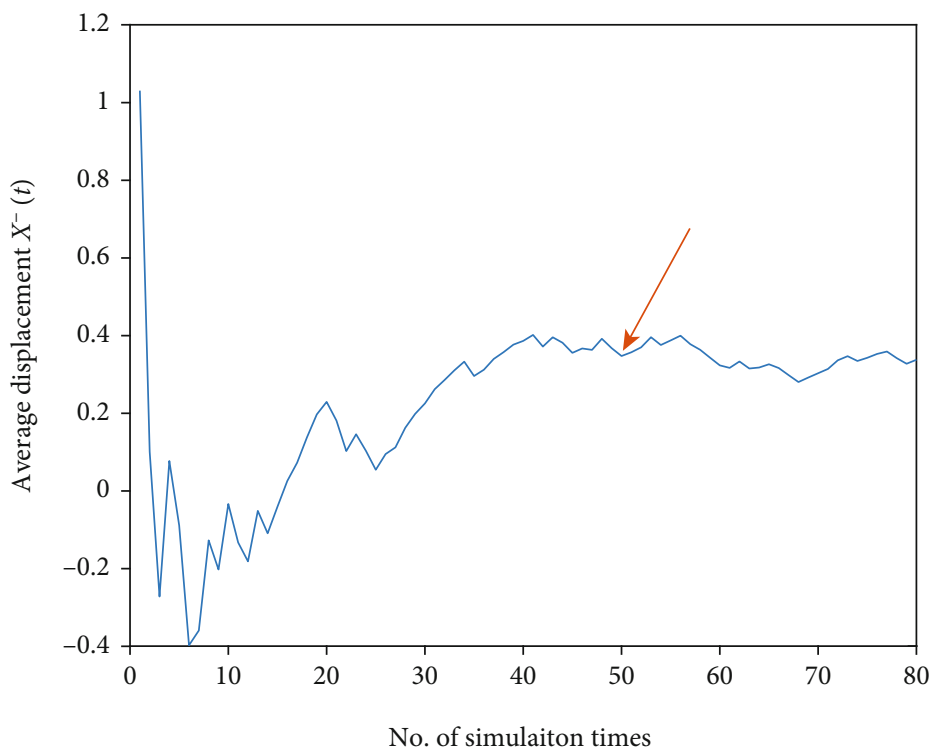

FIGURE 2: The relationship between the average displacement and the number of simulation time at a certain time. $x$-axis is the number of simulation time; $y$-axis is the average displacement.

2.4. The Lévy Noise. Lévy noise is also well known as the alpha stable noise, which was proposed by Lindberg Lévy. Since neither the distribution function nor the probability density function of Lévy noise has an explicit expression, the distribution of Lévy noise is usually expressed by a characteristic function as follows:

$\varphi(t)= \begin{cases}\exp \left[-\sigma^{\alpha}|t|^{\alpha}\left(1-i \beta \frac{2}{\pi} \operatorname{sign}(t) \log |t|\right)+i \mu t\right], & \alpha=1, \\ \exp \left[-\sigma^{\alpha}|t|^{\alpha}\left(1-i \beta \frac{2}{\pi} \operatorname{sign}(t) \tan \frac{\pi \alpha}{2}\right)+i \mu t\right], & \alpha \neq 1 .\end{cases}$
Here, $\alpha \in(0,2]$ is the stability index, $\beta \in[-1,1]$ is the skewness parameter, $\sigma>0$ is the scale parameter, and $\mu \in R$ is the shift.

\section{Analyses of the Effects of Different Noises on System Output}

We compare the system outputs driven by Gaussian white noise, harmonic noise, asymmetric dichotomous noise, and Lévy noise, which have certain guiding significance for stochastic resonance phenomenon driven by other noises, due to their wide applications.

3.1. The Simulation Number of Times. To obtain a stable state of the system responses, we need to avoid the randomness of 
TABLE 1: The common parameters for numerical simulation of Section 3.

\begin{tabular}{lccccccc}
\hline Parameter & Value & Parameter & Value & Parameter & Value & Parameter & Value \\
\hline$T$ & $1000 \mathrm{~s}$ & TestNum & 50 & $F s$ & 200 & $\theta$ & 0.03 \\
$\omega$ & $0.02 \mathrm{pi}$ & $\eta$ & 0.5 & $\Omega$ & 1 & $\Gamma$ & 1 \\
\hline
\end{tabular}

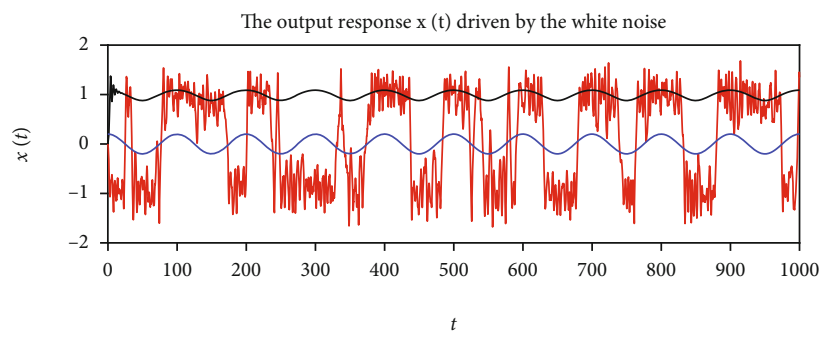

(a) The output response $x(t)$ driven by the white noise

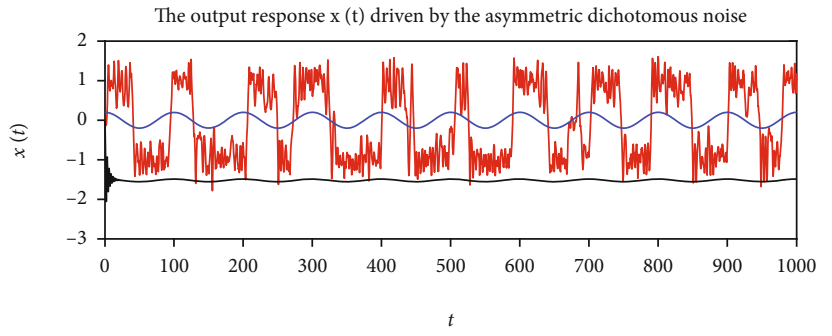

(c) The output response $x(t)$ driven by the asymmetric dichotomous noise

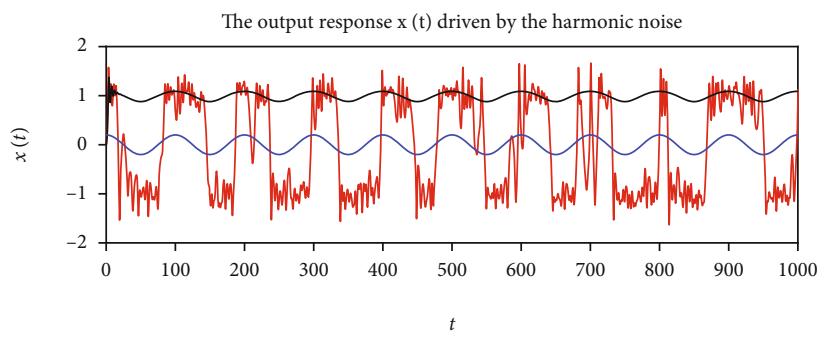

(b) The output response $x(t)$ driven by the harmonic noise

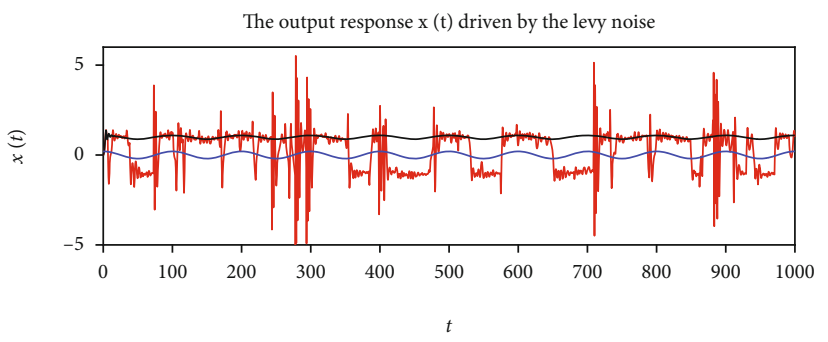

(d) The output response $x(t)$ driven by the Lévy noise

Figure 3: The system responses $x(t)$ for underdamped system under different noises. The values of the parameters for the numerical simulation are shown in Table 1. The blue line is for input sine signal. The black line is for $D=0$, and red line is for $D=0.07$.

the noises by considering the statistical average in numerical simulation. Therefore, we will determine the number of simulations in this paper by observing the relationship between the average particle displacement $\bar{X}(t)=\sum_{i=1}^{N_{i}} X(t) / N_{i}$ and the number of simulations $N_{i}$.

In Figures 1 and 2, it is obvious that the average displacement tends to be stable when the number of simulations is 50 , which indicates that when simulation time equals to 50 , it can reveal the general rule of the system output. Therefore, it is reasonable for us to use 50 simulations to reveal the rule of the noise-driven dynamical phenomena in the following simulation process.

Furthermore, without a special request, the common simulation parameters will be used in the following table:

3.2. The System Response Driven by Different Noises. We have compared the response of the system driven by Gaussian white noise, harmonic noise, asymmetric binary noise, and Lévy noise, respectively. The simulation values are set as Table 1.

Figure 3 shows the performance of the response of the periodic modulated underdamped system driven by different noises in the time domain, where Figure 3(a) is the situation driven by the white noise, Figure 3(b) is the situation driven by the simple harmonic noise, and Figure 3(c) is the situation driven by the asymmetric dichotomous noise. Figure 3(d) shows the situation driven by Lévy noise. Four kinds of noise can be found that they can cause approximately periodic transitions between two states. And we found that the jump frequencies of the four noise are close to the frequencies of the input periodic forces. In other words, under the simulation conditions with the same parameters as shown in Figure 3, the particles vibrate approximately synchronously with the input periodic force.

Besides, we found that the particles fluctuated in orbit around the noiseless input. At the same time, the output of the system driven by harmonic noise is obviously stronger than the other two damping effects. The vibration of particles is much stronger when they located in the two potential wells during the transition and then decreases significantly. The vibration in the potential wells is more stable than that of harmonic noise and asymmetric binary noise. Finally, the case driven by Lévy noise is the most special. Due to the impulse characteristic of Lévy noise, the particle displacement has great changes in some positions and then quickly returns to orbit.

3.3. The Stochastic Resonance of the System Driven by Different Noises. The power spectral density is the characteristic quantity of signal energy realization in the frequency domain, which reveals the characteristic of signal in the frequency domain. In the next sections, we will observe the influence of noise on system output through the power spectrum of system output at the frequency point of input periodic signal. 


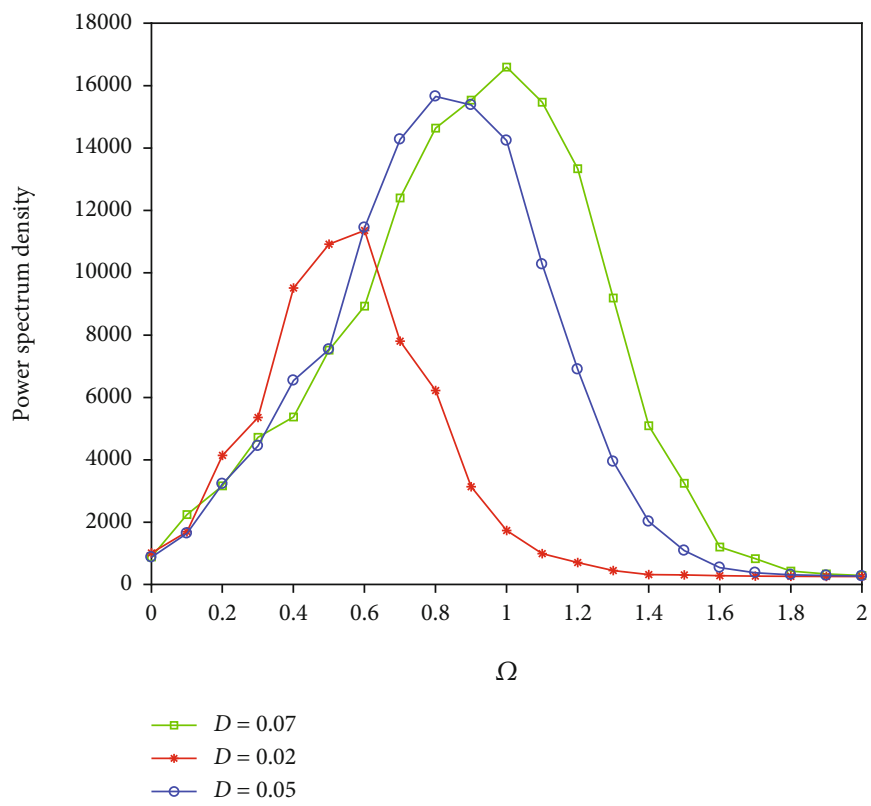

(a)

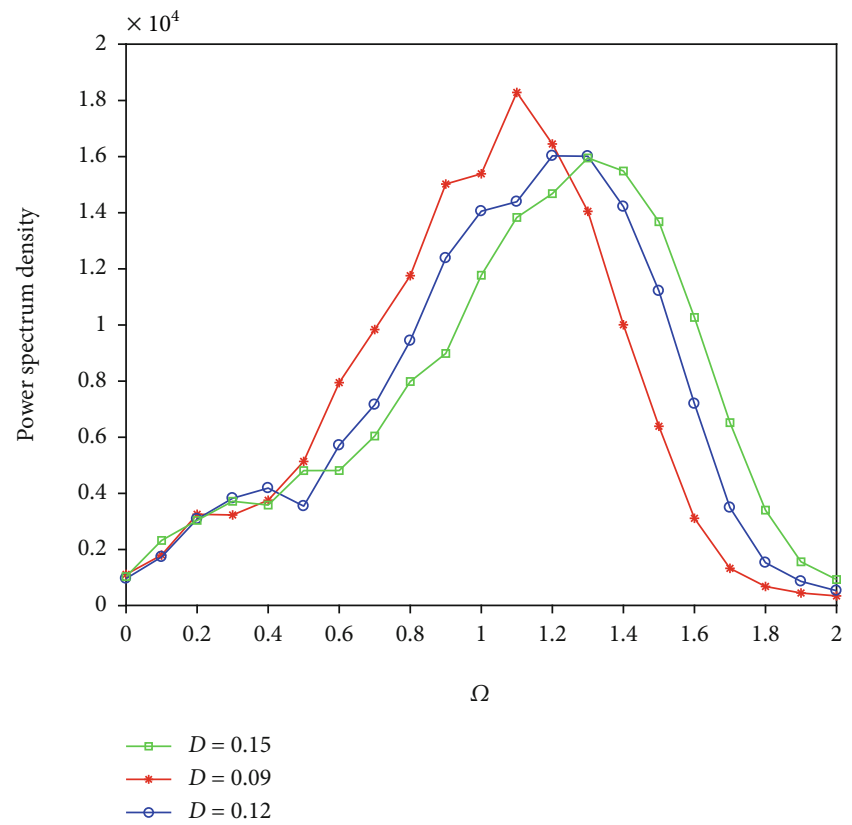

(b)

FIGURE 4: The relationship between $\Omega$ and system output PSD of undamped bistable system driven by the harmonic noises. (a) Input white noise intensity $D=0.02,0.05$, and 0.07 . (b) Input white noise intensity $D=0.09,0.12$, and 0.15 .

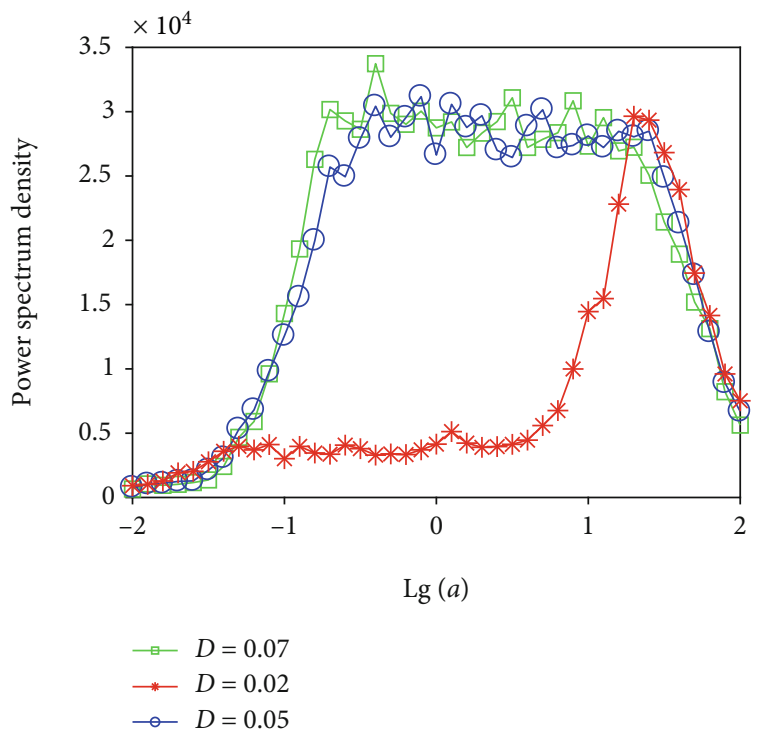

(a)

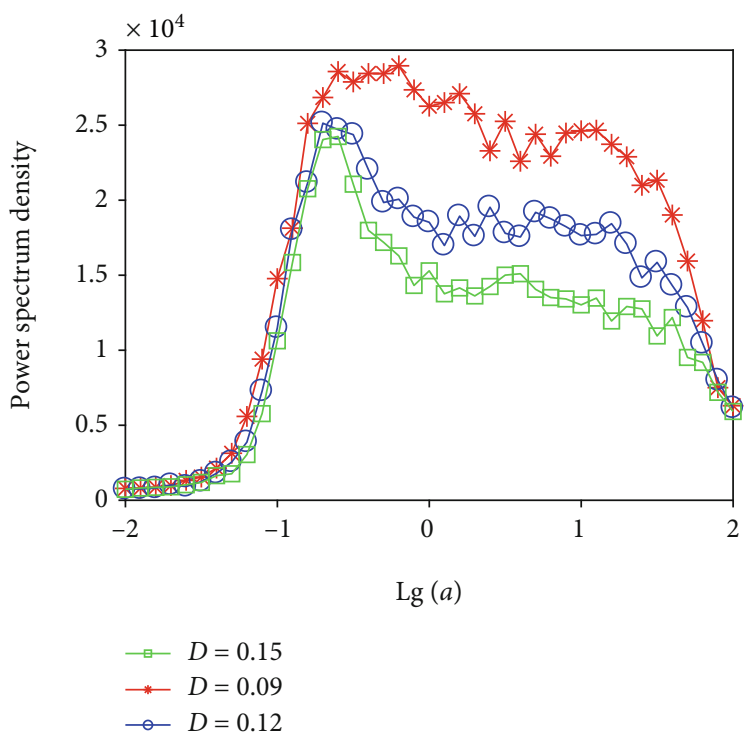

(b)

FIgURE 5: The relationship between $\lg (a)$ and system output of undamped bistable system driven by asymmetric dichotomous noise. (a) The output when input white noise intensity is $D=0.02,0.05$, and 0.07 . (b) The output when input white noise intensity is $D=0.09,0.12$, and 0.15 .

3.3.1. The System Driven by the Harmonic Noise. In this section, we did the numerical simulation by the EulerMaruyama method; the results are as follows.

Figure 4 shows the impact of $\Omega$ on system response PSD in harmonic noise (Equation (1)) model in the case of different noise intensities. With the increase of $\Omega$, the power spectrum density of the system response is increasing; then, it peaks and then goes down. There are obvious resonance peaks, and there is a random resonance phe- nomenon. Meanwhile, comparing the two pictures, it can be found that as the noise intensity increases, the power spectrum density of the system response also increases first and then decreases. The value of $\Omega$ corresponds to the peak that constantly moves to the right with the increase of noise intensity. Therefore, by adjusting the value of $\Omega$ in harmonic noise model, the stochastic resonance phenomenon can be induced by the undamped bistable system. 


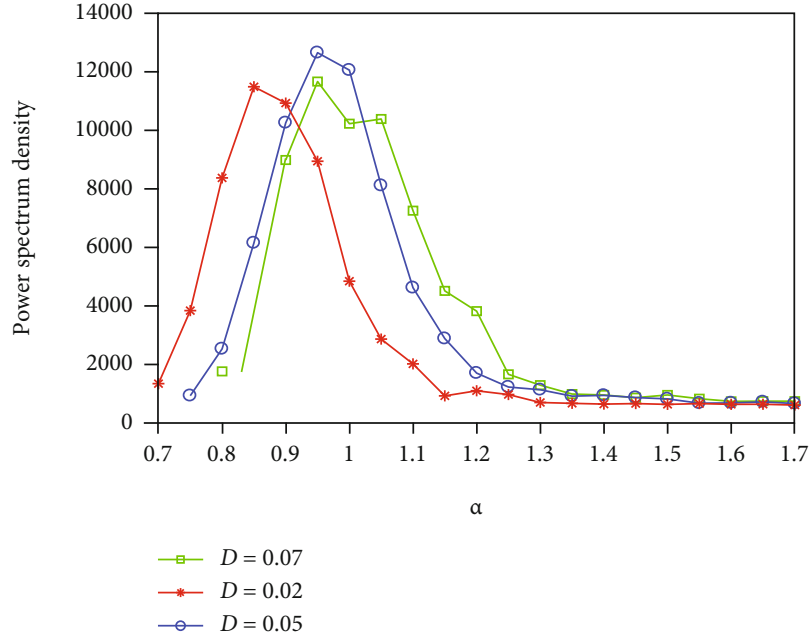

(a)

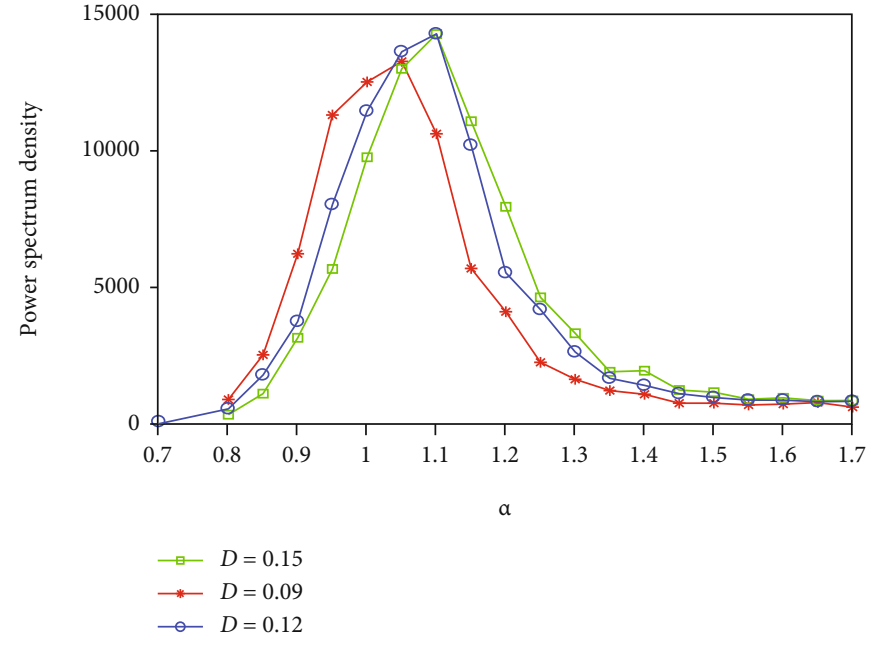

(b)

Figure 6: The relationship between characteristic exponent $\alpha$ and system output of undamped bistable system driven by Lévy noise. (a) The output when input is white noise intensity $D=0.02,0.05$, and 0.07 . (b) The output when input white noise intensity is $D=0.09,0.12$, and 0.15 .

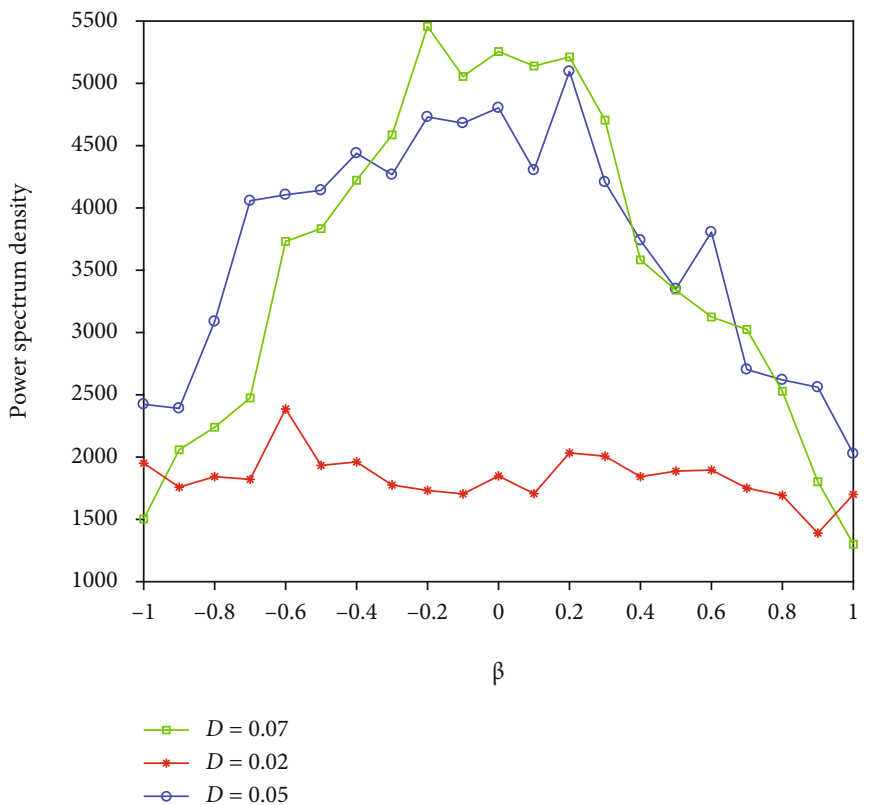

(a)

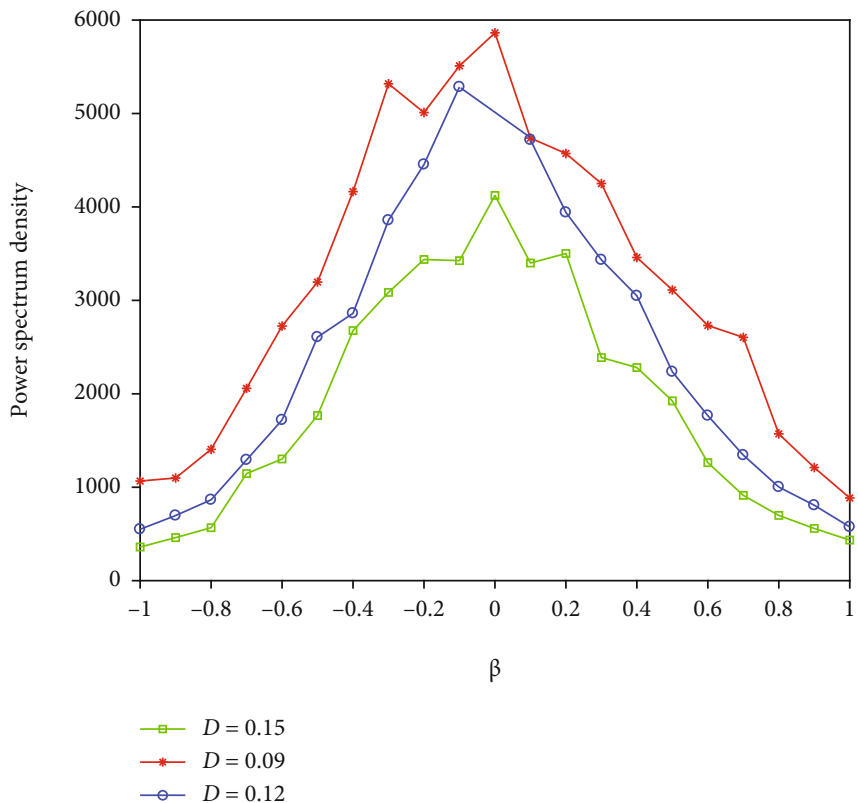

(b)

Figure 7: The relationship between symmetric parameter $\beta$ and system output of undamped bistable system driven by Lévy noise. (a) The output when input white noise intensity is $D=0.02,0.05$, and 0.07 . (b) The output when input white noise intensity is $D=0.09,0.12$, and 0.15 .

3.3.2. The System Driven by the Asymmetric Dichotomous Noise. We mainly focused on the relationship between the noise parameter $a$ and the system response PSD, for the two states of random telegraph noise are symmetrical. After the numerical simulation by the Euler-Maruyama method, the results are as follows.

Figure 5 shows the relationship between $a$ and system output of undamped bistable system PSD driven by asymmetric dichotomous noise with $b$ fixed. We find that there is stochastic resonance in this system. When it has low noise intensity, the resonance peak of power spectral density in system responding is narrower. With the increase of noise intensity, the width of the resonant peak is relatively stable, while the height of the resonant peaks goes from low to high.

3.3.3. The System Driven by the Lévy Noise. Due to the complex form of Lévy noise, it is difficult to apply the EulerMaruyama method and Milstein method to its numerical simulation. Therefore, we will use the fourth-order RungeKutta method for numerical simulation, to investigate the control effect of each parameter on stochastic resonance.

In Figure 6, we find there is stochastic resonance in the relational graph. With the increase of the noise intensity, the resonant peak of the power spectral density curve is 


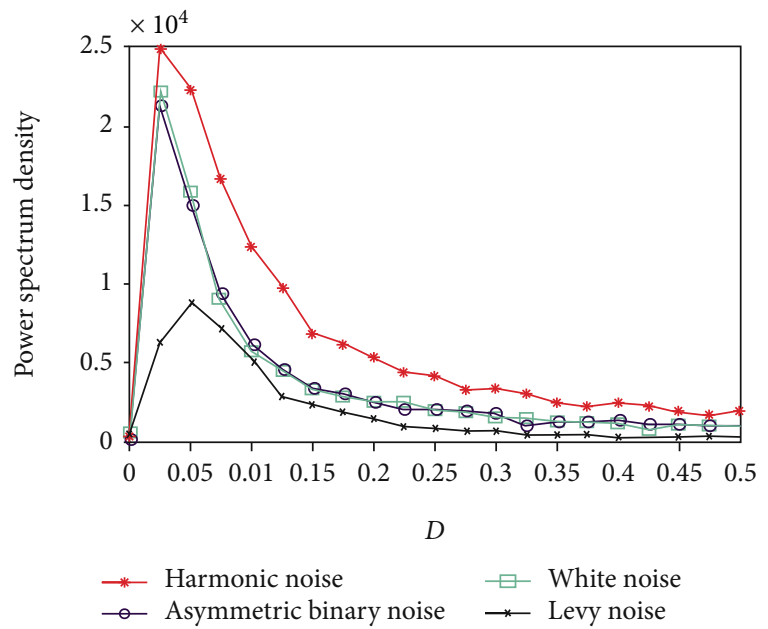

(a)

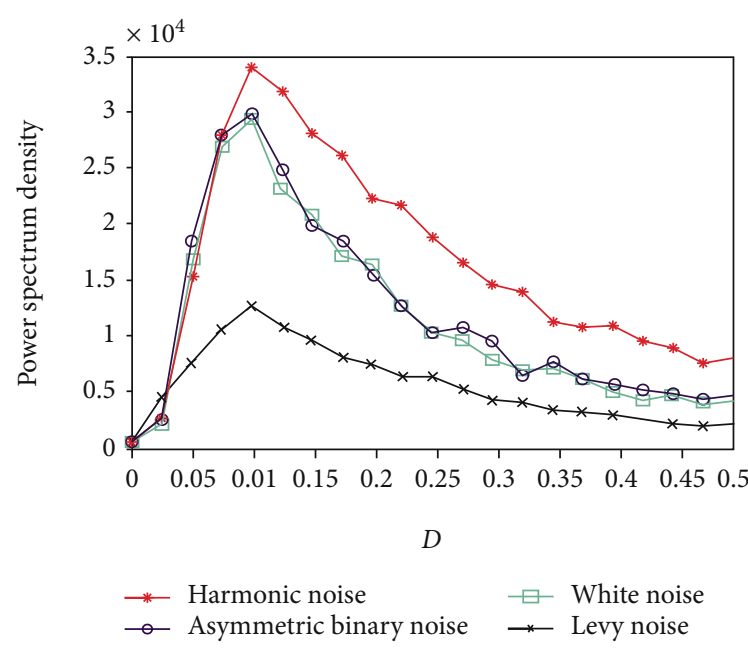

(c)

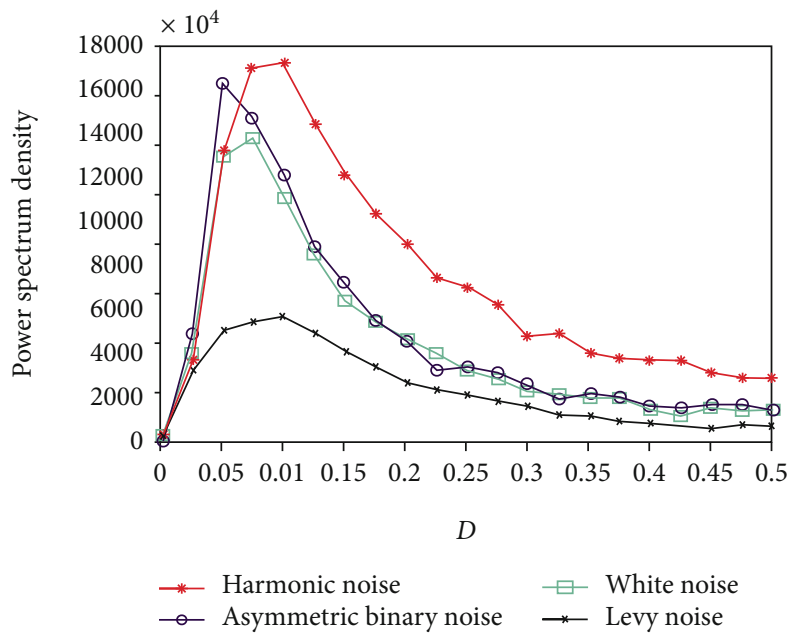

(b)

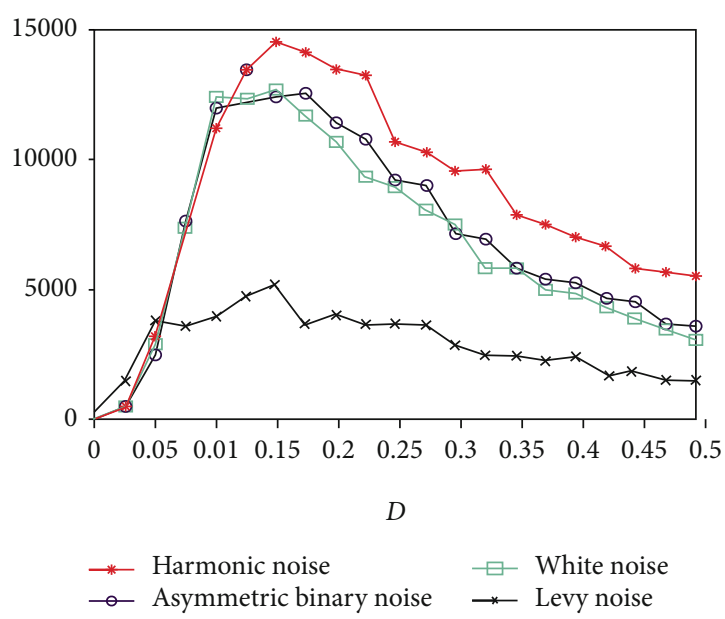

(d)

Figure 8: The relational graph between noise intensity $D$ and system output of undamped bistable system driven by four noise. (a) The output when the damping coefficient of the bistable system is $\eta=0.2$. (b) The output when $\eta=0.5$. (c) The output when $\eta=0.7$. (d) The output when $\eta=1.0$.

moving in the positive direction, that is, $\alpha$ reaching resonance peak grows with the increase of the noise intensity.

Comparing Figures 7(a) and 7(b) in Figure 7, we found that the stochastic resonance occurs in this system. With the increase of the noise intensity, the peak value of the resonant peak of the power spectral density curve increases and then decreases. However, the position of the peak is almost unchanged. Hence, by controlling the symmetric parameter $\beta=0$, we lead to phenomenon of the stochastic resonance of the symmetric parameters of the undamped bistable system driven by Lévy noise.

3.3.4. The Comparison of Different Noises on Stochastic Resonance. It can be found that four kinds of noise can induce the approximate periodic transition between the two states. And the frequency of the four types of jumps is close to the frequency of the input periodic forces. That is, the particle is almost synchronized with the periodic force of the input under the same parameter simulation condition. The particles are all moving up and down in the direction of the noiseless input. At the same time, we also find that the output of system output generated by harmonic noise is significantly stronger than that of other two. The vibration of the particle in two potential well is stronger when the transition occurs and then significantly decreases. Compared with that, the harmonic noise and asymmetric noise are more stable in the potential well. The case driven by Lévy noise is the most special one. Because of the pulse characteristic of steady noise, the displacement of particles varies greatly in some places and then rapidly returns to orbit.

In Figure 8, the noise intensity $D$ can be used to guide the occurrence of random resonance. With the undamped double steady-state damping coefficient increasing, the resonant peaks of noise intensity $D$ are constantly moving to the right. It indicates that damping has an inhibitory effect on random forces. In four kinds of noise, the output power spectral density of the harmonic noise at the signal frequency point is highest, and that from white noise and asymmetric 
dichotomous noise takes second place. And that from steady noise is the lowest. When harmonic noise, asymmetric dichotomous noise, and white noise are at low noise intensity, they are very close to the spectral density of the periodic signal. The output power spectrum of the system output in the undamped bistable system is basically coincident with white noise and asymmetric dichotomous noise. The resonant peak of the stochastic resonance of the Lévy noise and the harmonic noise drive is very close to the resonant peak driven by the other two noises. It can be found that the noise type has little influence on the resonance peak of the undamped bistable system on the noise intensity $D$.

\section{Conclusion}

In this paper, we mainly study the control effect of four kinds of noise (white noise, harmonic noise, asymmetric dichotomous noise, and Lévy noise) on undamped bistable system. The random resonance phenomenon is generated by adjusting the noise parameters. Since the harmonic noise can be generated by the white noise through the resonance subsystem, we can change the properties of harmonic noise by controlling the parameters of the resonance subsystem and then we can control stochastic resonance. In this paper, the control effect of harmonic noise in undamped bistable system is studied, and the similar random resonance phenomenon is found in the undamped bistable system.

The four kinds of noise used in stochastic resonance research are simulated by numerical algorithms. Here, Euler numerical algorithm and Milstein numerical algorithm are based on stochastic differential equation and fourth-order Runge-Kutta algorithm is based on ODE. The main object of the analysis is the time domain diagram and the power spectral density diagram of the system output.

In this paper, it has indicated that in the undamped bistable system, the stochastic resonance can be controlled by the harmonic noise generated by the resonance subsystem. The four kinds of noise (white noise, harmonic noise, asymmetric dichotomous noise, and Lévy noise) can be used to change the noise parameters and control stochastic resonance under certain conditions. For these four kinds of noise, the power spectrum density of the system output is very close to the horizontal position of the resonant peak of the noise. That is, the noise type has little influence on the horizontal position of the resonant peak of stochastic resonance after full optimization.

\section{Data Availability}

The data used to support the findings of this study are included within the article.

\section{Conflicts of Interest}

The authors declare that there is no conflict of interests regarding the publication of this paper.

\section{Acknowledgments}

This work is supported by the Fundamental Research Funds for the Central Universities (Grant No. 2682018CX65).

\section{References}

[1] R. Benzi, A. Sutera, and A. Vulpiani, "The mechanism of stochastic resonance," Journal of Physics A: Mathematical and General, vol. 14, no. 11, pp. L453-L457, 1981.

[2] P. Hanggi, P. Jung, C. Zerbe, and F. Moss, "Can colored noise improve stochastic resonance," Journal of Statistical Physics, vol. 70, no. 1-2, pp. 25-47, 1993.

[3] L. Gammaitoni, P. Hänggi, P. Jung, and F. Marchesoni, "Stochastic resonance," Reviews of Modern Physics, vol. 70, no. 1, pp. 223-287, 1998.

[4] Z. Qiao, Y. Lei, and N. Li, "Applications of stochastic resonance to machinery fault detection: a review and tutorial," Mechanical Systems and Signal Processing, vol. 122, pp. 502536, 2019.

[5] Y. Jia, S.-N. Yu, and J.-R. Li, "Stochastic resonance in a bistable system subject to multiplicative and additive noise," Physical Review E, vol. 62, no. 2, pp. 1869-1878, 2000.

[6] S. L. Lu, Q. B. He, and F. R. Kong, "Effects of underdamped step-varying second-order stochastic resonance for weak signal detection," Digital Signal Processing, vol. 36, pp. 93-103, 2015.

[7] R. Ray and S. Sengupta, "Stochastic resonance in underdamped, bistable systems," Physics Letters A, vol. 353, no. 5, p. 364, 2005.

[8] Y. F. Guo, F. Wei, B. Xi, and J. G. Tan, “The instability probability density evolution of the bistable system driven by Gaussian colored noise and white noise," Physica A: Statistical Mechanics and its Applications, vol. 503, pp. 200-208, 2018.

[9] D. Barik, P. K. Ghosh, and D. S. Ray, "Langevin dynamics with dichotomous noise; direct simulation and applications," Journal of Statistical Mechanics-Theory and Experiment, vol. 2006, no. 3, Article ID P03010, 2006.

[10] Z. Qiao, Y. Lei, J. Lin, and S. Niu, "Stochastic resonance subject to multiplicative and additive noise: the influence of potential asymmetries," Physical Review E, vol. 94, no. 5, article 052214, 2016.

[11] C. J. Wang, F. Long, P. Zhang, and L. R. Nie, "Controlling of stochastic resonance and noise enhanced stability induced by harmonic noises in a bistable system," Physica A Statistical Mechanics \& Its Applications, vol. 471, p. 288, 2016.

[12] X. Y. Luo, "Stochastic resonance in neuronal network motifs with Ornstein-Uhlenbeck colored noise," Mathematical Problems in Engineering, vol. 2014, Article ID 902395, 7 pages, 2014.

[13] G. K. Er, H. T. Zhu, and V. P. Lu, "Nonzero mean PDF solution of nonlinear oscillators under external Gaussian white noise," Nonlinear Dynamics, vol. 62, no. 4, p. 743, 2010.

[14] G. Zhang, Y. Song, and T. Q. Zhang, "Stochastic resonance in a single-well system with exponential potential driven by Levy noise," Chinese Journal of Physics, vol. 55, no. 1, pp. 85-95, 2017.

[15] Z. Gingl, L. B. Kiss, and F. Moss, "Non-dynamical stochastic resonance: theory and experiments with white and arbitrarily coloured noise," EPL (Europhysics Letters), vol. 29, no. 3, p. 191, 1995.

[16] Y. J. Shen, Y. F. Guo, and B. Xi, "Steady state characteristics in FHN neural system driven by correlated non-Gaussian noise 
and Gaussian noise," Acta Physica Sinica -Chinese Edition, vol. 65, no. 12, 2016.

[17] L. Zeng, B. Xu, and J. Li, "Dynamical properties of subdiffusion in the bistable system with aperiodic inputs," Physics Letters A, vol. 361, no. 6, pp. 455-459, 2007.

[18] A. Neiman and L. Schimansky-Geier, "Stochastic resonance in bistable systems driven by harmonic noise," Physical Review Letters, vol. 72, no. 19, pp. 2988-2991, 1994.

[19] B. Ahmad, J. J. Nieto, and A. Alsaedi, "A study of nonlinear Langevin equation involving two fractional orders in different intervals," Nonlinear Analysis Real World Applications, vol. 13, no. 2, pp. 599-606, 2012.

[20] R. L. Honeycutt, "Stochastic Runge-Kutta algorithms.1. White noise," Physical Review A, vol. 45, no. 2, pp. 600-603, 1992.

[21] R. L. Honeycutt, "Stochastic Runge-Kutta algorithms. II. Colored noise," Physical Review A, vol. 45, no. 2, pp. 604-610, 1992.

[22] W. Rumelin, "Numerical treatment of stochastic differential equations," SIAM Journal on Numerical Analysis, vol. 19, no. 3, pp. 604-613, 1982. 